\title{
OPTIMAL ALLOCATION OF RENEWABLE DISTRIBUTED GENERATION WITH LARGE PENETRATION TO MITIGATE POWER QUALITY ISSUES
}

\section{Divya S}

Assistant Professor, Department of Electrical and Electronics, GSSS Institute of Engineering \& Technology for Women, Mysuru, India

\section{Dr. S.B. Shivakumar}

Professor, Department of Electrical and Electronics, Rao Bahadur Y. Mahabaleswarappa Engineering College, Bellary, India

\section{Dr. T Ananthapadmanabha}

Principal, NIE Institute of Technology, Mysuru, India

\begin{abstract}
The intervention of DG's in the electrical network has increased remarkably which has led to the requirement of optimally allocating them in the system. They should be allocated with an optimal size and appropriate locations such that they increase the system performance, reduce losses as well as to obtain better voltage profile. In this paper the nature inspired algorithm based intelligent technique called modified shuffled frog leap algorithm is used to obtain the optimal location of the renewable distributed generation. Here analysis is carried out on full load and heavy load and constant power load model is considered for the case of heavy load where drastic changes in the test system can be observed. Under this condition the optimal placement and selection of type of DGs with their penetration level is the important factor for consideration. The enhancement of power quality is achieved by nullifying the effect of the voltage sag at the affected busses by using the indices based on the variations in voltage. The entire analysis is carried out on standard IEEE 33bus test system.
\end{abstract}

Key words: Renewable Distributed Generation, Large Penetration, Optimal Location, Sag. 
Cite this Article: Divya S, Dr. S.B. Shivakumar and Dr. T Ananthapadmanabha, Optimal Allocation of Renewable Distributed Generation with Large Penetration To Mitigate Power Quality Issues, International Journal of Electrical Engineering \& Technology (IJEET) 10(3), 2019, pp. 10-15.

http://iaeme.com/Home/issue/IJEET?Volume=10\&Issue=3

\section{INTRODUCTION}

In the power system, it is required that the operator maintains the voltage within the limits for each customer bus. There are many standards which have been proposed for satisfactory voltage profiles in distribution systems. It is proposed that the voltage variations in the distribution system must stay in the range of $-13 \%$ to $7 \%$, which is generally maintained at $6 \%$ in the utilities. Introduction of the DGs in the networks enhances the voltage profile by changing the path of power flow.

With the enhancement of the technology, power quality issues have been under concern leading to the change in the nature of electric loads, due to their nonlinearity, they lead to disturbance in the voltage waveform. Power quality issues will increase the disturbance in the system. Any disturbance occurring will result in huge financial loss and consequent loss of productivity. Hence, some steps must be taken to mitigate these issues to obtain a good quality of power.

When the system voltage reduces to 10 to $90 \%$ of the RMS value of actual voltage for 0.5 second to 1 minute is called as the sag. This is caused mainly due to faults or overloading due to large motors. The voltage sag results in malfunctioning of protective devices which leads to an interruption in the processes and tripping.

\section{PROBLEM FORMULATION}

Power quality can be improved by optimally allocating the available distributed generation sources using the shuffled frog leap algorithm. The inclusion of DG in an appropriate way will give a better profile of the voltage and reduce the system losses, hence improving the system performance by reducing the power quality problems.

\subsection{Power Quality assessment Indices}

The system performance will be depending on the quality of the power supplied and in various cases it is specified by using System Average rms Frequency Index (SARFI) [3]., it presents the average number of RMS variations over the assessment period to the customer served, and it calculates the number of busses effected by voltage sag by crossing the lower limit of 0.9.p.u. If the limit is fixed to $90 \%$ irrespective of the duration it is represented as SARFI-90.

$$
\text { SARFI }=\frac{\text { Total number of buses experiencing voltage sag (Nsag) }}{\text { Total number of customers served }}
$$

And the improvement index of the same is represented as

$$
S A R F I I=\frac{\left(S A R F I_{\text {with } D G}\right)}{S A R F I_{\text {without } D G}}
$$

Where,

SARFI $=$ System Average rms Frequency Index

SARFII $=$ System Average rms Frequency Improvement Index

$\mathrm{SARFI}_{\text {withoutDG }}=$ System Average rms Frequency Improvement Index without DG 


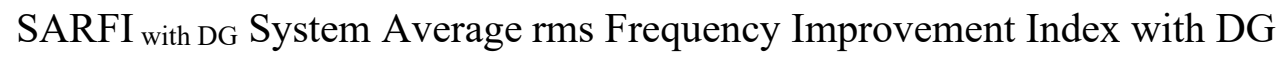

Nsag $=$ Total number of buses experiencing voltage sag.

$N_{\text {sag }}=\sum_{i=1}^{N_{\text {bus }}}\left\{\begin{array}{c}1 \text { if } 0.1 \text { pu }<V_{i}<0.9 p . u \\ 0 \quad \text { otherwise }\end{array}\right.$

Where, $\mathrm{N}_{\text {sag }}$ corresponds to busses which fall below the lower limit under the heavy loaded condition. Nbus corresponds to number of buses.

Hence, the following fitness functions are to be minimized to improve the quality of power.

$$
\begin{aligned}
& F 1=\min (\text { Nsag }) \\
& F 2=\min (\text { SARFI })
\end{aligned}
$$

\subsection{Methodology}

This section shows the results obtained after the execution of SFLA on IEEE 33 bus, For the network considered the following three cases are analyzed and for each case, three scenarios are considered.

Case-1: Allocation of only Solar DG units

Case-2: Allocation of only wind powered DG units

Case-3: Allocation of both solar and wind powered DG units

Scenario -1: With penetration level of $15 \%$

Scenario -2: With penetration level of $30 \%$

Scenario -3: With penetration level of $50 \%$

Case -4: Allocation of only solar DG unit under heavy load condition

Case -5: Allocation of only wind powered DG unit under heavy load condition

Case -6: Allocation of both solar and wind powered DG unit under heavy load condition

Scenario -1: Integration of single DG unit

The allocation of DG is based on the population of shuffled frogs with its will decide the location of DG. With respect to the power requirement and the deviation in voltage, the bus with maximum voltage deviation is chosen as the best location with the corresponding size depending on power losses.

\subsection{Modified Shuffled Frog-Leaping Algorithm}

MSFL algorithm is a meta-heuristic method which finds the global optimal solution by performing a heuristic search. It is an evolution process in which information is exchanged globally carried by individual's memes and population. Thus it combines the benefits of the local search and global search. MSFLA mainly based on the behavior of groups of frogs searching for the location that has maximum amount of available food. The MSFLA involves a population of possible solutions defined by a set of virtual frogs. This set of virtual frogs is partitioned into subsets known as memeplexes. The memeplexes can be perceived as a set of parallel frog cultures attempting to reach same goal. Frog leaping improves an individual frog and enhances its performance towards the goal. Within each memeplex each frog holds different ideas and the idea of each frog can be used to infect the ideas of other frogs. The process of passing information between the frogs of memeplex is known as, 'local search' or ' memetic evolution step'. After a defined number of memetic evolution steps, the virtual frogs are shuffled and reorganized, so that the quality of memeplex is improved. Shuffling enhances 
the memetic quality after infection and ensures the cultural evolution towards a particular interest. The process of evolution and shuffling are repeated until a required convergence is reached.

\subsection{Solution Methodology}

\section{The sequential steps are as follows [7]:}

1. Begin

2. Generate random population of P solutions (Frogs)

3. for each individual P: calculate fitness

4. Sort the population $P$ in descending order of their fitness

5. Divide P into m memeplexes;

6. for each memeplex: Determine the best and worst frogs

7. Improve the worst frog position.

8. Repeat for a specific number of iterations, End

9. Combine the evolved memeplexes

10. Sort the population $\mathrm{P}$ in descending order of their fitness

11. Check if termination $=$ true

12. End

\section{RESULTS AND DISCUSSIONS}

This method is applied to the standard IEEE 33 bus system operated at $12.66 \mathrm{kV}$ level of voltage and consists of 33 busses, 32 branches, with $3715 \mathrm{~kW}$ and $2300 \mathrm{kVar}$ of power respectively as shown in Figure.3.1. The base case load flow is obtained where the power losses without the connection of the DG's are $202.68 \mathrm{~kW}$ and $143.22 \mathrm{kVAr}$ respectively.

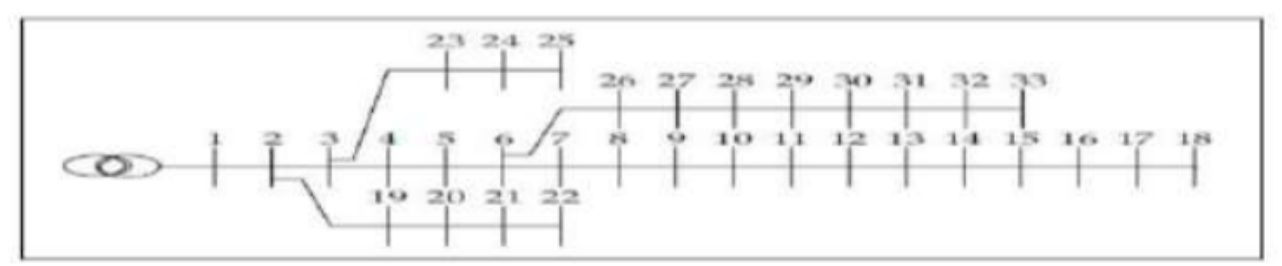

Figure 3.1 Single diagram of IEEE 33 bus test system

Table 3.1 Case 1,2,3 for scenario 3 with 15\% penetration with three DGs

\begin{tabular}{|l|c|c|c|}
\hline \multicolumn{1}{|c|}{ 3 } & Solar DG & Wind DG & Both Solar and Wind \\
\hline 1: Optimal location & Bus 17 & Bus 29 & Bus 33 \\
\cline { 2 - 4 } 2: Optimal size in MW & Bus 07 & Bus 07 & Bus 06 \\
\cline { 2 - 4 } & Bus31 & Bus31 & Bus31, \\
\hline 3: Base case power losses & 202.68 & 202.68 & 202.68 \\
\hline 4: Power losses with DG in & 152.7 & 164.77 & 123.62 \\
\hline 5: Power loss reduction in & 49.83 & 37.82 & 78.97 \\
\hline 6: power loss reduction in & 24.6 & 18.67 & 30.98 \\
\hline \multirow{2}{*}{$\begin{array}{l}\text { 7: Minimum voltage } \\
\text { without DG in p.u. }\end{array}$} & Bus 18 & Bus 18 & Bus 18 \\
\hline \multirow{2}{*}{$\begin{array}{l}\text { 8: Minimum voltage with } \\
\text { DG in p.u. }\end{array}$} & 0.91309 & 0.91309 & 0.91309 \\
\cline { 2 - 4 } & Bus 14 & Bus 18 & Bus 18 \\
\hline
\end{tabular}


Optimal Allocation of Renewable Distributed Generation with Large Penetration To Mitigate Power Quality Issues

Table 3.2. Case 1,2,3 for scenario 2 with $30 \%$ penetration with two DGs

\begin{tabular}{|l|c|c|c|}
\hline \multicolumn{1}{|c|}{ Particulars } & Solar DG & Wind DG & Both Solar and Wind \\
\hline 1: Optimal location & Bus 32 & Bus 2, & Bus 6, Bus33, \\
\hline 3: Base case power losses in kW & 202.68 & 202.68 & 202.68 \\
\hline 4: Power losses with DG in kW & 128.15 & 142.23 & 103.7 \\
\hline 5: Power loss reduction in kW & 74.53 & 60.37 & 98.86 \\
\hline 6: power loss reduction in \% & $36.88 \%$ & 29.80 & 48.81 \\
\hline 7: Minimum voltage without DG in p.u. & Bus 18 & Bus 18 & Bus 18 \\
\cline { 2 - 4 } & 0.91309 & 0.91309 & 0.91309 \\
\hline 8: Minimum voltage with DG in p.u. & Bus 30 & Bus 18 & Bus 18 \\
\cline { 2 - 4 } & 0.96872 & 0.96308 & 0.9249 \\
\hline
\end{tabular}

Table 3.3 Case 1,2,3 for scenario 1 with 50\% Penetration with single DG

\begin{tabular}{|l|c|c|c|}
\hline \multicolumn{1}{|c|}{ Particulars } & Solar DG & Wind DG & Both Solar and Wind \\
\hline 1: Optimal location & Bus 7 & Bus 31 & Bus 32, bus 15 \\
\hline 2: Base case power losses in kW & 202.68 & 202.68 & 202.68 \\
\hline 3: Power losses with DG in kW & 108.08 & 121.61 & 61.23 \\
\hline 4: Power loss reduction in kW & 94.6 & 81.07 & 141.45 \\
\hline 5: power loss reduction in \% & 46.67 & 40.01 & 69.7 \\
\hline 6: Minimum voltage without DG in p.u. & Bus 18 & Bus 18 & Bus 18 \\
\cline { 2 - 4 } & 0.91309 & 0.91309 & 0.91309 \\
\hline \multirow{2}{*}{ 7: Minimum voltage with DG in p.u. } & Bus 18 & Bus 18 & Bus 18 \\
\cline { 2 - 4 } & 0.94526 & 0.92515 & 0.9398 \\
\hline
\end{tabular}

Table 3.4 Case 4,5,6 for scenario 1 with $100 \%$ penetration single DG

\begin{tabular}{|l|c|c|c|}
\hline \multicolumn{1}{|c|}{ Heavy load } & Solar DG & Wind DG & Both Solar and Wind \\
\hline 1: Optimal location & Bus 29 & Bus 30 & Bus 31, bus6 \\
\hline 2: Base case power losses in $\mathrm{kW}$ & 559.66 & 559.66 & 559.66 \\
\hline 3: Power losses with DG in $\mathrm{kW}$ & 322.16 & 381.28 & 206.69 \\
\hline 4: Power loss reduction in $\mathrm{kW}$ & 237.5 & 178.38 & 352.97 \\
\hline 5: power loss reduction in \% & 42.43 & $31.87 \%$ & $63.06 \%$ \\
\hline \multirow{2}{*}{$\begin{array}{l}\text { 6: Minimum voltage without DG in } \\
\text { p.u. }\end{array}$} & Bus 18 & Bus 18 & Bus 18 \\
\hline \multirow{2yyy}{*}{ 7: Minimum voltage without DG in p.u } & 0.85493 & 0.85493 & 0.85493 \\
\cline { 2 - 4 } & Bus 18 & Bus 18 & Bus 18 \\
\cline { 2 - 4 } & 0.8853 & 0.87733 & 0.90211 \\
\hline Sag exposed area \% before DG & 53.33 & 53.33 & 53.53 \\
\hline Nsag, Before DG placement & 16 & 16 & 16 \\
\hline Sag exposed area \% after DG & 15.15 & 18.18 & 3.03 \\
\hline Nsag, After DG placement & 5 & 6 & 1 \\
\hline SARFI,Before DG placement & 0.0051233 & 0.0051233 & 0.0051233 \\
\hline SARFI,after DG placement & 0.001601 & 0.001701 & 0.0011 \\
\hline SARFII & 0.68750 & 0.80286 & 0.78529 \\
\hline
\end{tabular}


Table 3.1,3.2 and 3.3 shows the different penetration levels at $15 \%, 30 \%$ and $50 \%$ respectively, of solar, wind and hybrid solar with wind distributed generators. The losses are reduced the maximum value with hybrid combination for the same penetration level. The losses with solar distributed generation are obtained to be less compared to wind distributed generators. Table 3.4 shows the results obtained by integrating Renewable DGs at optimal buses with 100 percent penetration levels using MSFLA under the heavy load condition. The renewable distributed generators are able to manage the heavy load on the system as obtained in Table3.4 with single DG integration and with hybrid power generation.

\section{CONCLUSION}

In this paper, the allocation of DG with optimal size and location is done using a natureinspired algorithm called modified shuffled frog leap algorithm. This is a newly developed intelligent technique which gives better results. Here, optimal placement of DG is done based on the moment of frogs in search of food. The analysis is carried out on the IEEE 33 bus test system for different scenarios by considering the renewable distributed generators. It is evident that the percentage losses in the system are reduced and minimum voltage at the bus is increased with the hybrid generation of both solar and wind. During heavy load, the DG will compensate the entire additional load by nullifying voltage sag at the affected nodes.

\section{REFERENCES}

[1] Afzalan E., Taghikhani M.A., 'DG Allocation and sizing using MSFLA to reduce losses and improve voltage profile in distribution network', Journal of sustainable energy vol. 3, no. 3, September, 2012.

[2] P Kayal, C.K. Chanda, 'Placement of wind and solar based DGs in distribution system for power loss minimization and voltage stability improvement', International Journal of Electrical Power and Energy Systems, Vol. 53, pp.795-809,2013.

[3] H.Shareef, Aa Ibrahim, N Salman A Mohamed, Power Quality And Reliability Enhancement In Distribution Systems Via Optimum Network Reconfiguration By Using Quantum Firefly Algorithm, Ep\&Es 58(2014) 160-169, Elsevier

[4] S Kaur, G Kumbhar, J Sharma, 'A MINLP technique for optimal placement of multiple DG units in distribution systems', International Journal of Electrical Power and Energy Systems, Vol. 63, pp.609-617, 2014.

[5] M Esmaili, E Chaktan Firozjaee, H Shayanfar, 'Optimal placement of distributed generations considering voltage stability and power losses with observing voltage-related constraints', International Journal of Applied Energy, Vol. 113, pp.1252- 1260,2014

[6] S Kaur, G Kumbhar, J Sharma, 'A MINL P technique for optimal placement of multiple DG units in distribution systems', International Journal of Electrical Power and Energy Systems, Vol. 63, pp.609-617,2014.

[7] R Ishak, A Mohamed, A N. Abdalla, M Wanik, 'Optimal placement and sizing of distributed generators based on a novel MPSI index', International Journal of Electrical Power and Energy Systems, Vol. 60, pp.389-398,2014.

[8] Mohamed Imran A, Kowsalya M, Optimal Size and Siting of Multiple Distributed Generators In Distribution System Using Bacterial Foraging Optimization, S\&Ec 15(2014) 58-65, Elsevier

[9] Power Quality Indices and Objectives, 18th International Conference On Electricity Distribution, Germain Beaulieu- Geert Borloo, Cired, Ccigre Wg 36.07

[10] optimal allocation of dispatchable and non-dispatchable dg units in distribution networ International Journal of Electrical Engineering and Technology (IJEET) (Vol.8, No. 6) Publication Date: 2017-12-30 